\title{
Buchbesprechungen - Book Reviews - Livres nouveaux
}

Méthodes subjectives et objectives d'appréciation des Caractères Organoleptiques des denrées alimentaires (Journées scientifiques du Centre National des Etudes et Recherches sur la Nutrition et l'Alimentation), vol. 1, 606 pages (Centre National de la Recherche Scientifique, Paris 1966). L'étude du gout et de la flaveur des denrées alimentaires et des mets qu'elles servent à préparer n'a pas d'intérêt seulement pour les gourmets mais aussi son importance pour le nutritionniste et le diététicien est considerable, car ces qualités «organoleptiques» ont une influence certaine sur Гappétit, le bon fonctionnement des organes digestifs et finalement sur le comportement psychique des individus. L'analyse qualitative et plus encore quantitative en est extrêmement delicate.

II convient tout d'abord d'étudier les modalités sensorielles et stimuli intéressés (olfaction, gustation, facteurs chimiques et mécaniques comme la consistance, etc.) et d'apprécier Tintensité des sensations, leur qualité, leur tonalité affective. II faut y ajouter les interactions olfacto-

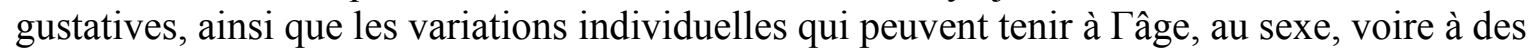
facteurs génétiques et surtout à Гédu-cation ou Гapprentissage. C'est ce qu'expose Le Magnen, dans son chapitre intro-ductif. Ensuite Mme Aschkenasy-Lelu étudie le role des caractérisations sensorielles et des propriétés nutritives des aliments dans la regulation du comportement alimentaire: quel peut être le mécanisme qui regie à court terme la grandeur ou le rythme des repas, nous Гignorons encore. Deux paraissent devoir être soulignés: Гimportance de la dimension affective des sensations gustatives et olfactives et de leur puissance de motivation - et d'autre part Гinterdépendance étroite de Гingestion alimentaire et de la couverture du besoin d'eau. Enfin la regulation de Гingestion calorique paraît subordonnée à la quantité et à la qualité des protéines ingérées.

Messereau étudie les méthodes statistiques appliquées au test des caractères organoleptiques (tests d'identification, de classement et de preference par 2 ou plusieurs produits, tests de coherence et d'homogénéité) et montre Гintérêt des notions qu'on en peut recueillir, tout en insistant sur les difficultés et les reserves que comporte cette approche mathématique d'un domaine aussi subjectif.

Depledt expose les principes généraux des méthodes d'analyse subjective des flaveurs, selon qu'il s'agit d'essais de recherches ou de fabrication à contrôler, le choix des échantillons, le materiel, la formation de comités de dégustation, les preuves et enfin les résultats qu'on en peut espérer. Tranchant décrit ensuite les possibilités de l'analyse des flaveurs par la chromatographie en gazeuse, technique d'une efficacité remarquable, bien que ne permettant pas d'aboutir à une definition precise des arômes et flaveurs étudieés.

Après les chapitres les Preferences d'une population de consommateurs (Mata-lon) et sur la Rhéologie des denrées alimentaires (Mocquot) la deuxième partie de Touvrage est consacrée aux Problèmes particuliers que posent les boissons, la bière, la viande, les corps gras, les produits laitiers, le pain, les conserves de fruits et legumes, les épices et aromates, le cafe, les cacaos sous la plume de spécialistes éminents. 
La conclusion est donnée par Souverain qui envisage le statut juridique du problème, 1 'application pratique de $\Gamma$ appréciation des caractères organoleptiques par des laboratoires officiels et les experts et enfin les tendances actuelles en France.

Tous les exposes sont suivis de discussion qui permettent de completer et d'éclaircir cette considerable documentation que représente ce volumineux ouvrage de 606 pages. II s'agit là de la plus importante mise au point d'un problème très Buchbesprechungen - Book Reviews - Livres nouveaux 77 délicat, et assez mal connu de ceux qui s'occupent de $\Gamma$ alimentation de $\Gamma$ homme sain ou malade. Elle fait le plus grand honneur à Torganisateur de ces Journées, le Prof. Tebroine. $\quad$ E. Azerad Verhandlungen der Deutschen Gesellschaft für Yerdauungs- und StoEfwechselkrankheiten, XXIII. Tagung in Wien 1965. Herausgeber: G.A.Martini. S. KargerAG, Basel/New York 1967. 406 S. Preis sFr./DM 112.-.

Der von G. A. Martini, dem derzeitigen ständigen Geschäftsführer der Deutschen Gesellschaft für Verdauungs- und Stoffwechselkrankheiten, herausgegebene Band enthält sämtliche Hauptreferate und Vorträge, die auf der 23. Tagung der Gesellschaft in Wien vom 23. bis zum 25. September 1965 gehalten wurden. Das Buch gliedert sich in zwei Teile: Der erste Teil enthält die Hauptreferate über die Themengebiete «Der operierte Magen», «dasPostcholecystektomieSyndrom», «Die Colitis ulcerosa» und «Funktionelle Pathologie des Pankreas». Eine ausführliche Wiedergabe des Inhaltes der einzelnen Hauptreferate ist selbstverständlich im Rahmen einer Besprechung nicht möglich. Im allgemeinen enthalten die einzelnen Kapitel einen mehr theoretischen Beitrag, zum Beispiel die Biochemie der Magen-sekretion von Heinz oder das zur Mineralogie der Gallensteine von Moselbach oder Ganganomalien des Pankreas und ihre klinische Bedeutung. Diesen theoretischen Darstellungen schliessen sich dann klinische Referate unter besonderer Berücksich-tigung der röntgenologíschen, chirurgischen und internistischkonservativen Aspekte an. Hervorgehoben seien folgende Beiträge: Korrekturoperationen am Magen von Nissen. Dieses Kapitel ist insofern von Bedeutung, weil die Indikation zur Zweitoperation gerade von Internisten wohl eher zu spat gestellt wird. Die Aus-führungen zum Postcholecystektomie-Syndrom betonen einhellig, dass es mit der Bezeichnung nicht getan sei, sondern dass die Beschwerden nach der Cholecystekto-mie einer sorgfältigen Analyse und einer entsprechenden Behandlung bedürfen. -Die psychosomatischen Probleme der Colitis ulcerosa werden von Schäfer und Wallis im Kindesalter und von Jores bei den Erwachsenen dargestellt, wobei deutlich klar wird, dass sie nur einen Teilaspekt der Krankheit darstellen, allerdings ist dieser Teilaspekt bei der Behandlung der Patienten sehr wichtig. Der neueste Stand der internistischen Therapie wird von Demling referiert, von Stelzner die chirurgischen Massnahmen erörtert. Wichtig sind die angiographischen Bilder bei einer Colitis ulcerosa, weil mittels der Angiographie sehr oft eine exaktere Beurtei-lung über die Ausdehnung der Colitis möglich ist. Der zweite Abschnitt bringt die Vorträge, die auf der gleichen Gesellschaft gehalten wurden, wieder. Hier gibt es 5 Hauptthemen: «Der operierte Magen», «Ga-stroskopische Diagnostik», «Postcholecystektomie-Syndrom», «Stoffwechseler-krankungen» und «Die Funktionsdiagnostik des Magens», ferner ein Rundgespräch zum Thema «Colitis ulcerosa».

«Der operierte Magen» enthält Untersuchungen über den Kohlenhydratstoff-wechsel nach Magen-Operation, Untersuchungen zur Resorption von D-Xylose, ferner die für die Praxis sehr wichtigen Untersuchungen von F. Kuhxencordt und H. Bartelheimer über die Auswirkungen der Magenresektion auf das Skelett. Die Symptomenarmut der Osteopathie führt oft erst in fortgeschrittenen Stadien zu einer Diagnose, wenn bereits irreparable Schäden aufgetreten sind. 
Das Wissen um die Möglichkeiten einer Skelettveränderung nach Magenresektionen ist Voraussetzung für eine frühzeitige Erfassung und prophylaktische Behandlung. Weiter eineingeschlossen sind Beiträge über eine neue, sehr interessante Technik der totalen Magenplastik von Liesco, Bukarest. Die gastroskopische Diagnostik befasst sich im wesentlichen mit Erfahrungsberichten über die Fiberskop-Untersuchung. Auch die

78 Buchbesprechungen - Book Reviews - Livres nouveaux

in diesem Abschnitt dargelegten Vorträge über Postcholecystektomie-Beschwerden betonen nur die Bedeutung einer exakten Analyse. - Der Beitrag «Stoffwechsel» enthält Untersuchungen über den Curare-Diabetes, Veränderungen am Leberparen-chym bei Diabetes mellitus, Permeabilitätsuntersuchungen an der Leberoberfläche. Strohmeyer und Dölle weisen in ihrem Beitrag nach, dass der $\mathrm{pH}-$ Wert des Blutes bei der Diffusion von Ammoniak durch die BlutLiquor-Schranke von Bedeutung ist und somit eine Rolle spielt bei der Ausprägung des ammoniakalischen Lebercomas. Heinkel schildert seine Erfahrungen mit der Endoradiosonde zur pH-Messung im Magen. Als Vorteil der Methode wird neben der leichteren Durch-führbarkeit hervorgehoben, dass eine Kontrolle des $\mathrm{pH}$ beim Übergang der Endoradiosonde in den Darm nur mit diesem Messverfahren möglich ist. Von Weymann, Giessen, wird hervorgehoben, dass die Alkali-Testzeit bei derschlauchlosenpH-Mes-sung mit der Endoradiosonde erhebliche Schwankungen aufweisen kann, so dass diese Methode keine sichej·en Aussagen gestattet. Abschliessend sei noch einmal festgestellt, dass in dem Hauptreferat von Haus-Amen und Rick zur exokrinen Pankreasfunktion nach Cholecystektomie sich An-gaben über die Normalwerte der Chymotrypsin-, Trypsin-, Carboxypeptidase-, Amylase- und Lipase-Konzentration im Duodenalsaft nach Pankreasstimulation mit Sekretin und Cecekin linden. Angaben über entsprechende Normalwerte bei derartigen Untersuchungen sind aus der Literatur nur schwer zu erhalten.

Im Rahmen einer Buchbesprechung konnten nur einige der zahllosen Hauptreferate und Vorträge zitiert werden, wobei die Auswahl notwendigerweise subjektiv sein muss. Es lässt sich aber feststellen, dass die Hauptreferate und die Vorträge zu den Hauptthemen einen guten Überblick über die aktuellen Probleme der entsprechenden Gebiete vermitteln. K. Becker, Hamburg Karla Longree: Quantity food Sanitation. (John Wiley et Sons Ltd., London 1967). 397 p. Prix: Pfund $4.16 .0 \mathrm{~d}$.

Environ 78 millions de repas sont servis chaque jour au dehors de la maison aux USA. II est clair que la preparation culinaire massive de repas pour de très grandes collectivités se développe rapidement avec Tindustrialisation. Ce sont les precautions nécessaires pour éviter les intoxications bactériennes qui sont étudiées minuti-eusement et très pratiquement.

Les 7 premiers chapitres sont consacrés à la bactériologie des aliments sous toutes leurs formes: lait frais, en poudre, viande, charcuterie, poulet, ceufs, aliments con-gelés, surgelés, fumes, etc. . . On étudie ensuite la legislation américaine sur la bactériologie alimentaire, les standards et les méthodes d'étude; puis le role de Гor-ganisation hygiénique de Гensemble des salles de stockage, de la cuisine; les sources de contamination secondaires par Thomme, les insectes, la terre, Гair; les principes des contrôles du personnel et de Гéquipement; le role des divers types de cuisson pour détruire les contaminants; Гéducation du personnel aux divers echelons. Ce livre sera précieux aux vétérinaires, agents de la repression des fraudes, aux responsables de très grosses preparations d'alimentation collective. II est remarquable par sa documentation et par son caractère très pratique, bien que d'un haut niveau.

J. Trémolières, Paris 
M. R. Kare et 0. Maller: The Chemical Senses and Nutrition. (The Johns Hopkins Press, Baltimore 1967). 495 p. Price: Dollar 12.50.

Cet ensemble de rapports tente de relier les approches des psychologues, neuro-physiologistes à ceux des zoologistes et nutritionnistes pour réaliser une unite de ce facteur majeur comportement alimentaire, le gout.

Buchbesprechungen - Book Reviews - Livres nouveaux 79

Quatre questions sont abordées: les changements subis par les sens chimiques au cours de revolution, les interactions des sens chimiques avec les métabolismes, la nutrition et la physiologie de Torganisme. Comment peut-on experimenter sur tel comportement ? Comment les sens chimiques s'intègrent-ils avec les autres perceptions?

Les sens chimiques aussi vieux que la cellule vivante elle-même, suffisants pour régler le comportement des unicellulaires, ne sont que l'un des petits rouages du comportement alimentaire de Thomme, le plus élaboré des mammifères.

Sens chimique chez les invertébrés, ingestion alimentaire et sens chimique chez les vertébrés inférieurs, chez Thomme suivant sa constitution, son niveau alimentaire et facteur génétique dans la perception, constituent les 5 etudes de la premiere partie.

Les invertébrés, bien que les récepteurs soient déjà très complexes, offrent un materiel experimental comportant moins d'éléments. Les serpents paraissent suivre les traces en touchant souvent le sol avec leur langue et en la reportant dans la bouche. Le venin paraît être le traceur qui, depose sur la souris, permet au serpent de la suivre, alors qu'il sent très mal une souris non mordue par lui. Chez Thomme, Гimportance des sens chimiques décroît avec le développement de la vision et le fonctionnement de ces sens paraît très incapable de bien régler les ingesta. Les facteurs génétiques sont difficiles à étudier, car le niveau du métabolisme c'est-à-dire des ingesta, le niveau endocrinien interviennent également.

La 2ème partie est consacrée aux modifications de Tolfaction et de la gustation suivant les états métaboliques.

Les addisons non traités, le syndrome adrénogénital, la fibrose du pancreas s'accompagnent d'une énorme exaltation de Tolfaction et de la gustation que les cortisoniques suppriment. A Topposé il existe une diminution de la sensibilité dans le syndrome de Turner.

Les rats surrénalectomisés et hypothyroïdiens ont tous deux un appétit parti-culier pour le sel. Cet appétit est salvateur. Mais lorsqu'on élève le taux de secretion de Гaldostérone par le propylthiouracile, il y a également un appétit accru pour le sodium qui est dangereux. C'est la perception «amer» qui est plus modifiée par les hormones, surtout sexu-elles. Des regimes très bas, très élevés, ou présentant un déséquilibre vitaminique (par excès ou par défaut) dépriment tous Гappétit du rat qui préfère un regime sans pro-téine à un regime mal équilibré en aminoacides.

La 3ème partie étudie le role conjoint de Tapprentissage et du gout dans la regulation des ingesta. L'apprentissage, la domestication modifient les effets du gout. Le rat sauvage regie mieux ses ingesta sur ses dépenses et moins sur les gouts. La réponse des nerfs afférents du gout depend de la secretion salivaire, de $\Gamma$ état endocrinien.

Les relations des sens chimiques ont pu être analysées par les etudes sur rat con-ditionné à se nourrir directement par sonde gastrique. Le gout et $\Gamma$ olfaction sont essentiels pour identifier les aliments. Ils constituent une motivation importante pour chercher les aliments. Sur le rat qui n'a pas faim, ils sont les seuls stimuli pour manger. Ils ont done à la fois un role discriminant et affectif. Mais vis-à-vis d'une alimentation monotone, ce sont les motivations internes qui sont 
mises en jeu. Des rats nourris directement dans Гestomac contrôlent parfaitement leurs ingesta, les sensations digestives et générales sont done suffisantes.

C'est la premiere fois qu'un aussi large tour d'horizon est consacré à la gustation et à l'olfaction. Cette etude souligne que $\Gamma$ approche analytique d'un comportement permet de comprendre mais pas de prévoir. J. Trémolières, Paris 80 Buchbesprechungen - Book Reviews - Livres nouveaux

I. SzORAdy: Die klinische Bedeutung der Pantothensäure unter besonderer Berück-sïchtigung der Kinderheilkunde. Szegedi Orvostudomanyi Egyetem, Szeged 1967. 104 Seiten.

Die Broschüre erscheint im Rahmen der von der Medizinischen Universität Szeged herausgegebenen Schriftenreihen «Studia Medica Szegedinensia». Szorady, Direktor des Kinderspitals Szeged, befasst sich seit langem mit der klinischen Anwendung der Pantothensäure, so dass die vorliegende Schrift nicht nur eine Zusammenfassung des weitverstreuten Schrifttums darstellt, sondern auch zahlreiche eigene Befunde und Betrachtungen des Verfassers selbst enthält. Kurze einleitende Abschnitte sind der historischenEntwicklung, der Chemie, der Analytik und dem natürlichen Vorkommen der Pantothensäure gewidmet. Die natürlichen Pantothensäurequellen sind sehr mannigfaltig, doch hebt der Verfasser auch hervor, dass die Werte in den verschiedenen publizierten Tabellen in weiten Bereichen schwanken (eine definitive Aussage über die mit der tischfertigen Nahrung aufgenommenen Vitaminmengen lassen diese Tabellen dennoch nicht zu. Ref.). - Eigene Unter suchungen des Verfassers, die sich verständlicherweise auf Säuglinge und Kleinkinder beziehen, ergaben unterschiedliche Blutspiegelwerte, aber keine Korrelationen zu irgendwelchen klinischen Symptomen. Bei Frühgeborenen ist jedoch mit einem relativen Panthothensäuremangel zu rechnen, da sie ein mangelhaftes Azetylierungsvermögen besitzen, was möglicherweise aus diesem Defizit resultiert. So bleibt zu klären, ob eventuell ein kausaler Zusammenhang zwischen der erhöhten Sulfonamidempfindlichkeit, dem geringeren Azetylierungsvermögen und der Pantothensäureversorgung bei Frühgeborenen besteht. - Der Pantothensäureblutspiegel liegt bei Kindern im ersten Lebenshalbjahr etwa 50\% über demjenigen der Erwachsenen und pendelt sich erst im 2. Lebenshalbjahr auf Normwerte ein. Welche besondere Bedeutung diesem erhöhten Blutspiegel zukommt und welche Zusammenhänge mit speziellen Stoffwechselabläufen bestehen, bleibt noch offen. - In weiteren Abschnitten werden die Stoffwechselbeziehungen der Pantothensäure abgehandelt, wobei eigene Untersuchungen an gesunden Kindern keine nennenswerten Effekte auf die wichtigsten Stoffwechselfunktionen erkennen liessen (u. a. Serumproteine, Pyruvat, Serumelektrolyte, Hb, SGOT, BSG, 17-Ketosteroide). Tierversuche lieferten Hinweise für eine mögliche korrigierende Wirkung der Pantothensäure auf Enzymstörungen (Leber: Succinatdehydrogenase; Cytochromoxydase). Ein grösseres Kapitel ist der klinischen Anwendung der Pantothensäure sowohl in prophylaktischer als auch therapeutischer Hinsicht gewidmet. Die am besten untermauerten Indikationen sind der paralytische Ileus und die postoperative Darmatonie mit den Vorteilen der Atoxizität und fehlenden Nebenwirkungen der Pantothensäure. Weniger überzeugend ist die toxizitätsmindernde Wirkung gegenüber Antibiotica aus der Streptomyces-Reihe. Auf die Pantothensäure-Applikation bei Affektionen der Atemwege, bei Verbrennungen, zur Wundheilung sowie bei Magen- und Darmulcera wird mehrfach hingewiesen. Schliesslich schreibt der Verfasser der Pantothen 
säure gewisse antiallergische Eigenschaften zu, was er experimentell zu belegen versucht. $\mathrm{Ob}$ der Pantothensäure tatsächüch eine Strahlenschutzwirkung zu kommt, insofern die Lebensdauer ganzkörperbestrahlter Mäuse nach Pantothensäurevorbehandlung verlängert ist, müsste wohl an anderen Tierspezies unter gleichzeitiger Heranziehung biochemischer Parameter intensiver überprüft werden. - Alles in allem enthält die Schrift eine gute Übersicht über den Stand der PantothensäureForschung bis zum Jahre 1962, wobei das ältere Schrifttum sehr umfangreich berücksichtigt wird. Allein 672 Literaturzitate aus der weitverstreuten Literatur sind zusammengetragen. W. F. Körner, Basel 